\title{
Oral Presentation III
}

\section{Accuracy of Color Doppler Ultrasound combined with Elastography in the Diagnosis of Malignancy of Thyroid Nodules with Suspicious FNAB}

\author{
${ }^{1}$ Seyed Ziaeddin Rasihashemi, ${ }^{2}$ Ali Ramouz, ${ }^{3}$ Nassir Rostambeigi \\ ${ }^{1,2}$ Department of Cardiothoracic Surgery, Tabriz University of Medical Sciences, Tabriz, Iran \\ ${ }^{3}$ Department of Radiology, University of Minnesota, Minneapolis, Massachusetts, USA
}

\section{BACKGROUND AND AIMS}

Thyroid nodules occur in more than $50 \%$ of populations over 50 years, and only $5 \%$ of thyroid nodules are malignant. This study was aimed to evaluate Doppler ultrasonography (US) combined with elastography in the diagnosis of the malignant thyroid nodules with suspicious fine needle aspiration cytological (FNAC) results.

\section{METHODS}

From August 2012 to March 2013, 107 consecutive patients eligible for thyroid surgery enrolled in the study. All patients underwent FNAC study followed by conventional US, color Doppler US, and US elastography with a real-time instrument. Thyroid nodules ultrasonographic parameters were evaluated during conventional and color Doppler US study and elastography performed in order to calculate the strain index (SI) by dividing the strain value of the nodule by that of the peripheral normal parenchyma, prior to thyroidectomy.

\section{RESULTS}

Of 161 nodules in 107 patients, 76 (47.2\%) were benign lesions and 85 (52.8\%) were malignant. The overall Doppler US score depicted a sensitivity of $64 \%$ and specificity of $59 \%$. Using a cut of 2.905 , the sensitivity and specificity for elastography US were about 54 and $76 \%$ respectively, with positive predictive value of $71.8 \%$ and negative predictive value of $59.8 \%$. Evaluating quantitative elastography combined with Doppler US, out of 68 nodules with positive peripheral halo ring, 44 had elasticity $\geq 2.905$, which was statistically significant $(\mathrm{p}<0.001)$.

\section{CONCLUSION}

Quantitative elastography combined with Doppler US is more accurate in thyroid nodules diagnosis comparing to other methods and can limit the use of FNAC and the subsequent thyroidectomy in patients with nondiagnostic or unsatisfactory cytological findings.

\section{Analysis of Rising Incidence of Thyroid Cancer in Singapore \\ ${ }^{1}$ Jesse Shulin Hu, ${ }^{2}$ Wee Boon Tan, ${ }^{3}$ Khuan Kew Chow, ${ }^{4}$ Aizhen Jin, ${ }^{5}$ En Yun Lai, ${ }^{6}$ Rajeev Parameswaran \\ ${ }^{1}$ Department of General Surgery, Jurong Health Services, Singapore \\ ${ }^{2,6}$ Department of General Surgery, National University Health Services, Singapore \\ ${ }^{3-5}$ National Cancer Registry, Health Promotion Board, Singapore}

\section{BACKGROUND AND AIMS}

Thyroid cancer is the most common endocrine malignancy. The annual incidence of thyroid cancer is known to vary with the geographic area, age, and gender. In recent years, there has been reports of increasing incidence of thyroid cancer worldwide, which has been attributed to increase in the detection of micropapillary subtype. We sort to investigate if this holds true in Singapore.

\section{METHODS}

This is a retrospective analysis of thyroid cancers treated in Singapore between the years 1974 and 2013. The data was obtained from the National Cancer Registry and included patients treated in all hospitals in Singapore. Demographic data on gender, age, ethnicity, tumor subtype, and mortality is available for the said period while additional data on tumor size and treatment is available only from 2007 onward.

For all included cases, the overall incidence and mortality of thyroid cancer for each year from 1974 to 2013 were calculated. All rates were age adjusted to the world standard population.

\section{RESULTS}

The age-standardized incidence rate of thyroid cancer increased by 224\% (2.5 per 100,000 in 1974 to 5.6 per 100,000 in 2013). Thyroid cancer affected women more than men by a ratio of 3 to 1 . The increased incidence was predominantly seen in the Malays by $290 \%$ (2.1 per 100,000 in 1974 to 6.0 per 100,000 in 2013) as compared to the Chinese and Indians. 
The distribution of histological categories was as follows: $81.1 \%$ papillary, $12.1 \%$ follicular, and $6.8 \%$ others (medullary and anaplastic). The increase in the incidence of papillary subtype is mainly due to the increase in the diagnosis of smaller cancers (less than $5 \mathrm{~cm}$ ).

Although the incidence of thyroid cancer has increased since 1974, the mortality rate has remained stable.

\title{
CONCLUSION
}

The trend of increasing incidence of thyroid cancer seen in Singapore is similar to that in other parts of the world, but it is not attributed to an increase in diagnosis of the micropapillary subtype.

\section{Clinical Evaluation of Thyroid RFA for Benign Thyroid Nodules in Japan}

\author{
${ }^{1}$ Masahide Nakano, ${ }^{2}$ Nobuhiro Fukunari, ${ }^{3}$ Toru Nishikawa, ${ }^{4}$ Sadatsugu Aida \\ ${ }^{1-4}$ Department of Surgery, Showa University School of Medicine, Northern Yokohama Hospital, Kanagawa, Japan
}

\section{BACKGROUND AND AIMS}

For the last 10 years, radiofrequency ablation (RFA) for thyroid nodules has been reported and could be a candidate for new alternatives to the conventional treatments. We started RFA in 2007 as a clinical study in Japan. We describe our cases and clinical courses of benign thyroid nodules treated with RFA.

\section{METHODS}

We had 57 nodules from 47 patients (43 females and 4 males; mean age 51.2; (23-78 years), which consisted of 46 hyperplastic nodules and 11 AFTNs. Radiofrequency ablation was performed under careful ultrasound guidance, and the intraoperative ablated area was estimated with US contrast agents. Post-therapeutic changes were followed with blood test and imaging diagnosis periodically (US and CT). Nodule size (major axis), thyroid function, and serum thyroglobulin level were evaluated before and after treatment at $1,3,6,12$, and 36 months.

\section{RESULTS}

Radiofrequency ablation for benign thyroid nodule showed the shrinkage of the nodule size gradually and obvious decrease in the nodule size almost 3 months later. The maximum diameter of the nodules were $46.18 \pm 17.51 \mathrm{~mm}$ (15-106 mm) before RFA, $39.81 \pm 15.22,35.37 \pm 13.72,30.97 \pm 12.51,29.97 \pm 11.54$, and $27.77 \pm 13.86 \mathrm{~mm}$ at 1, 3, 6, 12, and 36 months respectively.

All of AFTNs showed a euthyroid state with normal TSH level. We had three cases of skin burn that cured with only observation, and there were no cases of severe complications like nerve damage or hematoma. Both therapeutic effect and thyroid function have been preserved in the cases followed over 1 year.

\section{CONCLUSION}

Thyroid RFA is a safe and powerful tool for the treatment of benign thyroid nodules.

\section{Analysis of Clinicopathological Factors of Bethesda III (FLUS/AUS) Results in Fine Needle Aspiration Cytology of Thyroid Nodules}

\author{
${ }^{1}$ Tiffany Rui Xuan Gan, ${ }^{2}$ Kee Yuan Ngiam, ${ }^{3}$ Min En Nga, ${ }^{4}$ Wendy Wong, ${ }^{5}$ Jeffrey Lum, ${ }^{6}$ Wee Boon Tan, ${ }^{7}$ Rajeev Parameswaran \\ ${ }^{1}$ Yong Loo Lin School of Medicine, National University of Singapore, Singapore \\ 2,4,6,7Endocrine Surgical Unit, National University Hospital, Singapore \\ ${ }^{3,5}$ Department of Pathology, National University Hospital, Singapore
}

\section{BACKGROUND AND AIMS}

Fine needle aspiration cytology (FNAC) has become the gold standard for preoperative diagnosis of malignant thyroid nodules. However, Bethesda Category III: Follicular Lesion of Undetermined Significance (FLUS) or Atypia of Undetermined Significance (AUS) continues to cause diagnostic and therapeutic dilemmas. To address these issues, this study will describe the pickup rate of thyroid malignancies at a tertiary referral center in Singapore from FNACs diagnosed as FLUS/AUS and assess the significance of demographic and ultrasonographic features in predicting malignant outcomes in this group.

\section{METHODS}

A retrospective search on the pathology laboratory computerized database was conducted to identify all thyroid FNACs between 2008 and 2014 diagnosed by the Bethesda system as category III - FLUS/AUS at a single tertiary referral center - the National University Hospital in Singapore. Patient demographics, follow-up data, and preoperative ultrasonography features were collected for these patients and correlated with the final histopathological diagnosis in those who underwent surgery. 


\title{
RESULTS
}

A total of 311 thyroid nodules between 2008 and 2014 were diagnosed as FLUS/AUS. Of these, 139 (44.7\%) underwent surgical excision. The final histology showed $28.1 \%$ were malignant. Malignancy rate for AUS was significantly higher at $41.3 \%$ than for FLUS at $15.8 \%$ with a p-value of 0.001 , suggesting Bethesda III nodules should be further sub-stratified into AUS and FLUS and managed accordingly. A total of $61.6 \%$ had a more definitive diagnosis after up to three repeat FNACs. Demographic and ultrasonographic features were not significant in predicting malignant outcomes in patients in this group.

\section{CONCLUSION}

The diagnostic sensitivity of thyroid malignancies at our institution with the cytology of FLUS / AUS was 28.1\%, with a significantly higher rate for AUS compared to FLUS. Repeat FNACs are clinically useful in attaining a more definitive diagnosis in more than half of the cases.

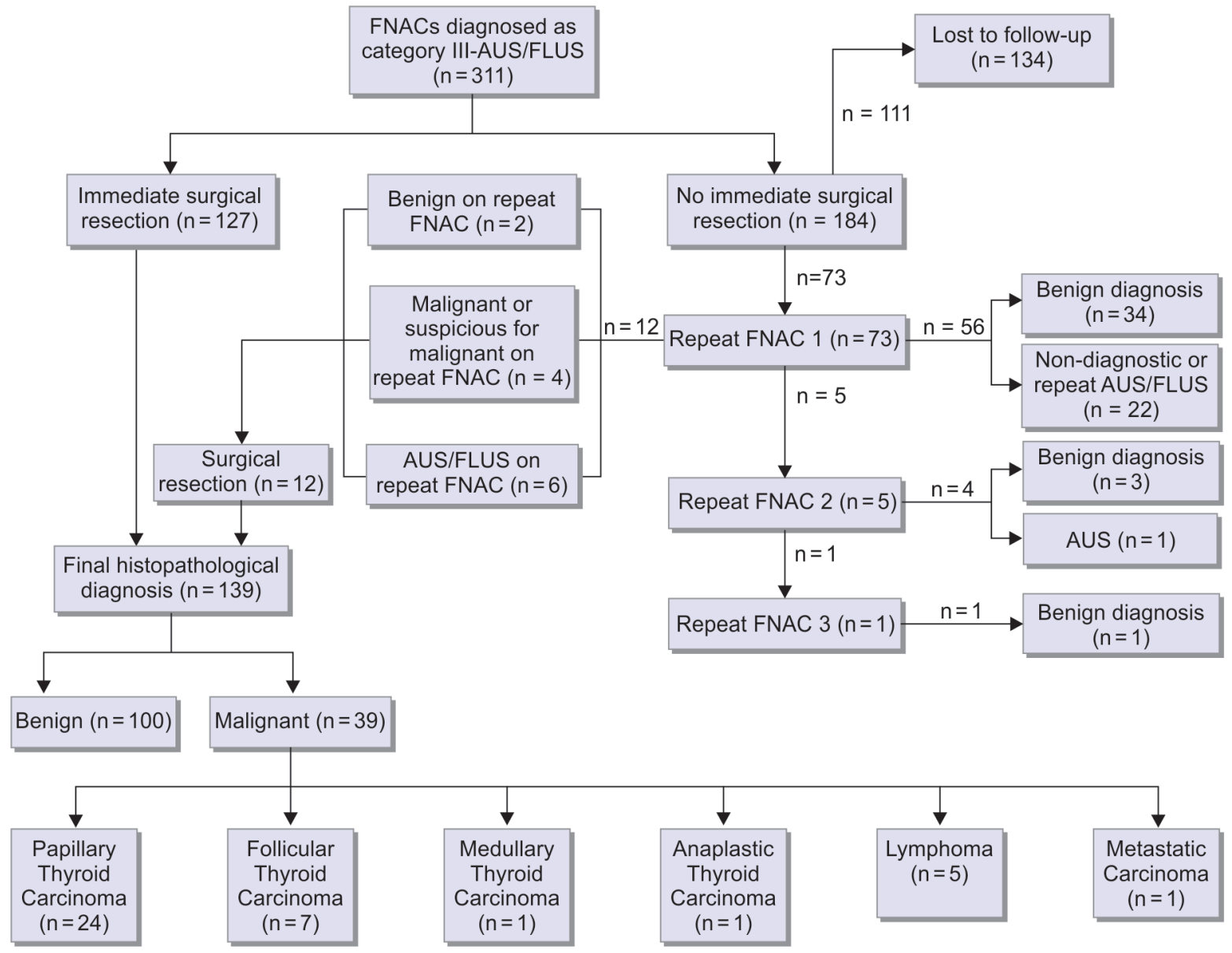

\section{Comparison of Treatment Outcome between Advanced Papillary Thyroid Carcinoma and early Thyroid Carcinoma: A Multicenter Study}

\author{
${ }^{1}$ Ki-Wook Chung, ${ }^{2}$ Yu-Mi Lee, ${ }^{3}$ June Young Choi, ${ }^{4}$ Kyu Eun Lee, ${ }^{5}$ Seok Mo Kim, ${ }^{6}$ Hang-Seok Chang \\ 1,2Department of Surgery, Asan Medical Center, Seoul, South Korea \\ ${ }^{3,4}$ Department of Surgery, Seoul National University Hospital, Seoul, South Korea \\ ${ }^{5,6}$ Department of Surgery, Gangnam Severance Hospital, Seoul, South Korea
}

\section{BACKGROUND AND AIMS}

Papillary thyroid carcinoma is the most common malignancy in Korea and usually demonstrates an indolent clinical course with an excellent survival rate. Therefore, minimizing surgical complications is as important as reducing the risk of recurrence. We gathered data of the patients who underwent thyroid surgery from three hospitals (Asan Medical Center, Gangnam Severance Hospital, and Seoul National University Hospital). We analyzed risk factors for developing complications and verified the correlation between the severity of the disease and the development of complications. 


\title{
METHODS
}

From 2004 to 2010, 17,569 patients underwent surgery due to papillary thyroid carcinoma. The cohort included 14,491 (82.5\%) females and 3,078 (17.5\%) males, with the mean age of $46.5 \pm 13.5$ years (5-109 years). The median follow-up period was 72 months (0-271 months). A total of 133 patients $(0.8 \%)$ showed distant metastasis at the initial diagnosis or within 12 months after the initial surgery. A total of 592 patients (3.4\%) developed recurrence during follow-up period. Of 11,496 patients who had total thyroidectomy, 3,530 (30.7\%) experienced postoperative hypoparathyroidism. Recurrent laryngeal nerve injury was accompanied in 214 patients $(1.2 \%)$, but only 44 patients $(0.4 \%)$ suffered permanent sequelae.

\section{RESULTS}

Age $\geq 45$ years, male gender, performing more extensive surgery (total thyroidectomy or cervical lymph node dissection), and TNM stage affected the development of complications in univariate analysis. However, after adjusting other values, advanced stage significantly related with the development of complications. Patients with T3 and T4 [odds ratio $(\mathrm{OR})=1.72 ; 95 \%$ confidence interval $(\mathrm{CI})=1.59-1.86 ; \mathrm{p}<0.001], \mathrm{N} 1(\mathrm{OR}=1.74 ; 95 \% \mathrm{CI}=1.60-1.88 ; \mathrm{p}<0.001)$, and stage III and IV $(\mathrm{OR}=1.70 ; 95 \% \mathrm{CI}=1.51-1.91$; $\mathrm{p}<0.001)$ had more complications than patients with early stage.

\section{CONCLUSION}

The treatment of advanced papillary thyroid carcinoma (PTC) was accompanied with higher rate of complication when compared with early PTC even with the same survival benefit.

\section{Costs for Treatment and Follow-up of Thyroid Cancer Increase according to the Severity of Disease}

\author{
${ }^{1}$ Soo Young Kim, ${ }^{2}$ Seok-Mo Kim, ${ }^{3}$ Bup-Woo Kim, ${ }^{4}$ Yong Sang Lee, ${ }^{5}$ Hang-Seok Chang, ${ }^{6}$ Cheong Soo Park \\ ${ }^{1-6}$ Department of Surgery, Gangnam Severance Hospital, Yonsei University College of Medicine, Seoul, South Korea
}

\section{BACKGROUND AND AIMS}

The increase in the diagnosis and treatment of thyroid cancer coincides with a growing national interest in cost-effective health care practices. The aim of this study was to provide an analysis of health care costs by the type of surgical extent of thyroid operation during 5 years.

\section{METHODS}

Patients who underwent thyroid operation for differentiated thyroid cancer in the year 2010 were identified from our institutional database. Patients underwent surgery according to their disease severity and were followed up for 5 years from 2010 to 2015. Costs for admission and outpatient clinic for thyroid cancer treatment were analyzed.

\section{RESULTS}

Outpatient clinic, admission, and overall cost increased with the extent of surgery. Patients who got only hemithyroidectomy incurred the lowest cost for outpatient follow-up, admission, as well as overall costs, and with the increasing extent of surgery, costs for follow-up and admission increased. Patients with total thyroidectomy, lateral neck node dissection, and mediastinal dissection showed highest costs (overall cost 5,766,759 $\pm 713,571$ vs 48,657,805 $\pm 16,957,932, p<0.001$ ).

\section{CONCLUSION}

Costs for admission and follow-up increased depending on the surgical extent and the disease severity respectively. Surgery at an early stage was most cost-effective when evaluated over 5 years.

\section{Risk Factors for Persistent/Recurrent Disease of Well-differentiated Thyroid Cancer in a Multiethnic Southeast Asian Population}

\author{
${ }^{1}$ Khee Chun Hiew, ${ }^{2}$ Anita Baghawi, ${ }^{3}$ Sarinah Basro, ${ }^{4}$ Normayah Kitan \\ ${ }^{1-4}$ Department of Breast and Endocrine Surgery, Ministry of Health, Hospital Putrajaya, Putrajaya, Malaysia
}

\section{BACKGROUND AND AIMS}

Well-differentiated thyroid cancers (WDTC) have a good prognosis in general; however, its recurrence increases morbidity and mortality. Identification of the risk factors for WDTC recurrence/persistence can improve clinical decision on aggressiveness 
in our management for the high-risk population. Among other well-studied risk factors, we aim to discover if the difference in ethnicity (Malay, Chinese, and Indian) contributes as a predictor for persistent/recurrent WDTC.

\title{
METHODS
}

This is a retrospective study that evaluated 275 consecutive patients operated for WDTC at Putrajaya Hospital, Malaysia, between January 2002 and November 2013, after excluding those with follow-up of less than 2 years. We studied various demographic, clinical, and histologic variables to determine their association with persistent/recurrent WDTC. The outcome was evaluated based on clinical, biochemical, and imaging data. Predictors that were significant on univariate analysis were subsequently evaluated with stepwise multivariate analysis.

\section{RESULTS}

The mean age of our patients was 44 years old; among them 102 (37.1\%) patients had persistent/recurrent disease within a median follow-up duration of 5.5 years. The risk factors found significant on univariate analysis were gender, size of tumor, extrathyroidal invasion, nodal involvement, and distant metastasis at presentation $(\mathrm{p}<0.05)$. Age has a weak association with recurrence/persistence $(\mathrm{p}=0.085)$; however, when included for multivariate analysis, it was significant $(\mathrm{OR}=1.03, \mathrm{p}=0.004)$, along with nodal involvement $(\mathrm{OR}=7.91, \mathrm{p}<0.001)$ and distant metastases $(\mathrm{OR}=4.430, \mathrm{p}<0.005)$.

\section{CONCLUSION}

Age, nodal involvement, and distant metastases are significant independent predictors for persistent/recurrent disease of WDTC in our multiethnic population, while ethnicity did not have any significant effect in this study.

\section{The Proportion of Patients with Papillary Thyroid Cancer Who can be salvaged from Total Thyroidectomy to Hemithyroidectomy by New ATA Guidelines}

\author{
${ }^{1}$ Musaed Fahad Rayzah, ${ }^{2}$ Jee Soo Kim, ${ }^{3}$ Jung-Han Kim, ${ }^{4}$ Jun-Ho Choe, ${ }^{5}$ Jung-Woo Woo, ${ }^{6}$ Inhye Park \\ ${ }^{1-6}$ Department of Surgery, Sungkyunkwan University School of Medicine, Samsung Medical Center, Seoul, South Korea
}

\section{BACKGROUND AND AIMS}

The current (2015) American Thyroid Association (ATA) guidelines recommend hemithyroidectomy for papillary thyroid cancer (PTC) patients with tumor size 1 to $4 \mathrm{~cm}$ in diameter that are unilateral, single, without extrathyroidal extension, and with no lymph node metastasis. We sought the proportion of patients who could have undergone hemithyroidectomy rather than total thyroidectomy according to new ATA guidelines under consideration of various clinicopathologic characteristics.

\section{METHODS}

We retrospectively reviewed the database of the thyroid cancer center of the Samsung Medical Center (SMC). Patients diagnosed from 1994 to 2014 with unilateral PTCs 1 to $4 \mathrm{~cm}$ in diameter, and who underwent total thyroidectomy, were included. By reference to the final histopathological results, we divided patients into three groups by tumor diameter (group I, tumor diameter 1.0-2.0 $\mathrm{cm}$; group II, 2.1-3.0 cm; and group III, 3.1-4.0 cm). The clinicopathologic features including extrathyroidal extension (ETE), tumor multiplicity, and lymph node (LN) status in each group of patients were analyzed. And we calculated the proportion of patients who could have undergone hemithyroidectomy rather than total thyroidectomy following the new 2015 ATA criteria for hemithyroidectomy. Statistical Analysis System (SAS) version 9.4 software was used for statistical analysis.

\section{RESULTS}

A total of 4,268 patients were eligible for this study; 3,393 (79.5\%) in group I, 683 (20.1\%) in group II, and 192 (5.7\%) in group III. A total of 718 patients (16.8\%) were suitable for hemithyroidectomy by 2015 ATA criteria: 598 (17.6\%) in group I; 99 (14.5\%) in group II; and $21(10.9 \%)$ in group III. Most of the patients $(83.2 \%)$ who had tumor size of 1 to $4 \mathrm{~cm}$ needed total thyroidectomy even after new criteria. The most common reasons for total thyroidectomy were extrathyroidal extensions $(n=3,062 ; 71.7 \%)$, followed by lymph node metastases $(n=2,521 ; 59.1 \%)$, and tumor multiplicity $(n=1,082 ; 25.4 \%)$.

\section{CONCLUSION}

Among patients with PTC between 1 and $4 \mathrm{~cm}$ in diameter, about 16\% could have undergone hemithyroidectomy rather than total thyroidectomy according to the 2015 ATA guidelines; however, a majority of patients still need total thyroidectomy due to ETE, LN metastasis, and tumor multiplicity. 


\title{
Quality of Life in Patients Who underwent Thyroidectomy compared to the General Population
}

\author{
${ }^{1}$ Hyeong Won Yu, ${ }^{2,3}$ Ah Reum An, ${ }^{4}$ Jin Wook Yi, ${ }^{5}$ Yong Joon Suh, ${ }^{3,6}$ Hyungju Kwon, ${ }^{3,7}$ Su-jin Kim \\ ${ }^{3,8}$ Young Jun Chai, ${ }^{3,9}$ June Young Choi, ${ }^{3,10} \mathrm{Ho}-$ Chun Choi, ${ }^{3,11}$ Belong Cho, ${ }^{3,12}$ Kyu Eun Lee \\ 1,4-7,12Department of Surgery, Seoul National University Hospital and College of Medicine, Seoul, South Korea \\ ${ }^{2,10,11}$ Center for Health Promotion and Optimal Aging, Seoul National University Hospital and College of Medicine, Seoul, South Korea \\ ${ }^{3}$ Cancer Research Institute, Seoul National University College of Medicine, Seoul, South Korea \\ ${ }^{8}$ Department of Surgery, Seoul National University Boramae Medical Center, Seoul, South Korea \\ ${ }^{9}$ Department of Surgery, Seoul National University Bundang Hospital, Seoul, South Korea
}

\section{BACKGROUND AND AIMS}

Preservation of quality of life (QoL) is an important therapeutic goal in patients with thyroid disease. The purpose of this study was to assess the impact of thyroidectomy on QoL compared to the general population while controlling the effect with propensity score matching.

\section{METHODS}

The QoL was retrospectively investigated in a patient who underwent thyroid surgery with thyroid disease and a control who visited the health screening center. The generic Short Form 12 questionnaire (SF-12) was adopted to measure the QoL. Mental component score (MCS) and physical component scores (PCS) were expressed as numeric values in SF-12. The electronic database Statistical Package for the Social Sciences (SPSS) version 22 was used for statistical analysis with 95\% confidence interval.

\section{RESULTS}

The QoL was retrospectively investigated in 105 patients with thyroid disease and 420 controls who visited the health screening center. The mean age of the patients and control were $49.18 \pm 12.6$ and $49.9 \pm 13.1$ years respectively. The male-to-female ratio was 15.0:85.0. On univariate analysis, it was found that two subtypes of physical component score in SF-12 are significantly different between patients and control group: Role Physical (SF-4, 38.5 vs 26.0\%, p = 0.011) and Role Physical (SF-5, 35.2 vs 20.2\%, $\mathrm{p}<0.001$ ), while three subtypes of mental component score in SF-12 were found in the following domains: Mental Health (SF-11, $3.07 \pm 1.49$ vs $2.61 \pm 1.32$, p = 0.002), Role Emotional (SF-7, 33.7 vs 20.7\%, p=0.005), and Social Functioning (SF12, $2.98 \pm 1.56$ vs $2.62 \pm 1.50, \mathrm{p}=0.03)$.

\section{CONCLUSION}

Thyroidectomy reduces the QoL in comparison to a healthy population considering both physical and mental components. Psychiatric consultation or supportive care may be helpful for patients with low value of mental scores. 